\title{
Implementation of Intelligent Green Energy Management System
}

\author{
Chao-Tung Yang ${ }^{1, *}$, Yin-Zhen Yan ${ }^{2}$, Yu-Chuan Shen ${ }^{3}$, Yun-Ting Wang ${ }^{4}$, Chao-Wei Huang ${ }^{5}$, Shuo-Tsung Chen ${ }^{6}$ \\ Dept. of Computer Science, Tunghai University, Taichung City, Taiwan
${ }^{1}$ ctyang@thu.edu.tw*; ${ }^{2}$ r541754175@gmail.com; ${ }^{3}$ s1991829@gmail.com; ${ }^{4}$ winnie3536@gmail.com; ${ }^{5}$ g02350034@thu.edu.tw; \\ ${ }^{6}$ shough33@thu.edu.tw \\ * corresponding author
}

\begin{abstract}
In this work, we mainly apply the cloud infrastructure (IaaS) and virtualization technology to provide the construction services of a green energy management system. First of all, we used MySQL Cluster database technology to build a data storage system which can solve the challenge of large demand. Digital electricity meter data and environmental information are collected efficiently and quickly in the proposed green energy management system. Next, a virtualized user-interface is provided by graphical presentation to facilitate data analysis. Finally, we control the electricity equipment to reduce Power Usage Effectiveness (PUE) and the overall power consumption target-based on this virtualized user-interface of the data analysis.
\end{abstract}

Keywords: Cloud infrastructure; Virtualization technology; MySQL cluster; Power saving; PUE;

\section{Introduction}

In recent years, with the rising of electricity cost and environmental awareness, how to save energy to achieve lower costs and the direction towards sustainable development has been a very important issue. The university's ITunits in our school, the server need to be provided the school various IT services which execute around 365 days a year to provide uninterrupted service. Although The power consumed ina single server is not large, but in the case of prolonged operation expenses will cause a lot of electricity.Therefore, for information on how to control and manage the cost of engine room is out the goal.

In this work, we mainly apply the cloud infrastructure (IaaS) and virtualization technology to provide the construction services of a green energy management system. First of all, MySQL Cluster database technology is adopted to build a data storage system which can solve the challenge of large demand. Accordingly, digital electricity meter data and the environment information are collected efficiently and quickly in the proposed green energy management system. Next, a virtualized user-interface is provided by a graphical presentation to facilitate data analysis. Finally, we control the electricity equipment to reduce Power Usage Effectiveness (PUE) and the overall power consumption target based on this virtualized user-interface of the data analysis.

The rest of the paper is as follows. In Section 2, we review background and related work. In Section 3, we introduce the proposed system design and implementation. Section 4 shows our experiment environment and results. In Section 5 , some conclusions are given.

\section{Background and Related Works}

\subsection{Background - OpenStack}

OpenStack began in 2010 as a cloud computing of joint project of Hackspace Hosting and NASA is an Infrastructure as a Service (IaaS) cloud computing project for public and private clouds. It is Apache license, free software and open source project. OpenStack includes a variety of modules which include computing module responsible for the deployment and management of virtual machine's features, Network module ensure that the services will not have problem when administrator deploy cloud services and network services. The storage module is responsible for scalable storage systems which Use the API to manage the network infrastructure systems. The final is Dashboard module, responsible for managing the previously described three modules. 
In this paper, we will use OpenStack to deploy its underlying architecture, because OpenStack deployment can help save costs,but also allow managers customize more value-added services, such as load balancing and monitoring tools.

\subsection{Background - Wireless Sensor Networks}

Wireless sensor networks are a lot of automatic devices distributed in space. It consists of a wireless communications network.In the wireless sensor network architecture, the concept of sensor design is based on small size, low cost, low power consumption, easy to build and has a distribution environmental sensing capabilities.In the beginning, "Smart Dust" [1], wireless sensor networks originally proposed by the University of California, Berkeley. Smart Dust not only control physical state of the environment by using sensors in different locations, but also has applications in military-related intelligence gathering.Now, because of MEMS and nanotechnology advance, the sensors constantly shrink volume, lightweight, carry positioning sensing node on the object. The various types of micro-sensor elements are combined with wireless transmission communication network, and large sensors are spread in the environment. This technology can provide the information you need at any time, the application can be reached in life today for the requirements of LOHASenvironment, including convenience, safety, comfort, energy saving, and has been widely used in various fields as environmental sensing and ecological health detection, traffic control, etc.

Wireless sensor networks [2] consist of three main areas: sensing, communications, computing. The key technologies include wireless data library technology, such as wireless sensor networks using queries, and used to other sensors and communications network technology. The basic structure of the sensing network system shows in Figure 1. First, a large number of Sensor Nodes placed to be sensed Sensor Field to collect data needed. And then by using Zigbee to send gather information from Wireless Data Collector back to the manager.

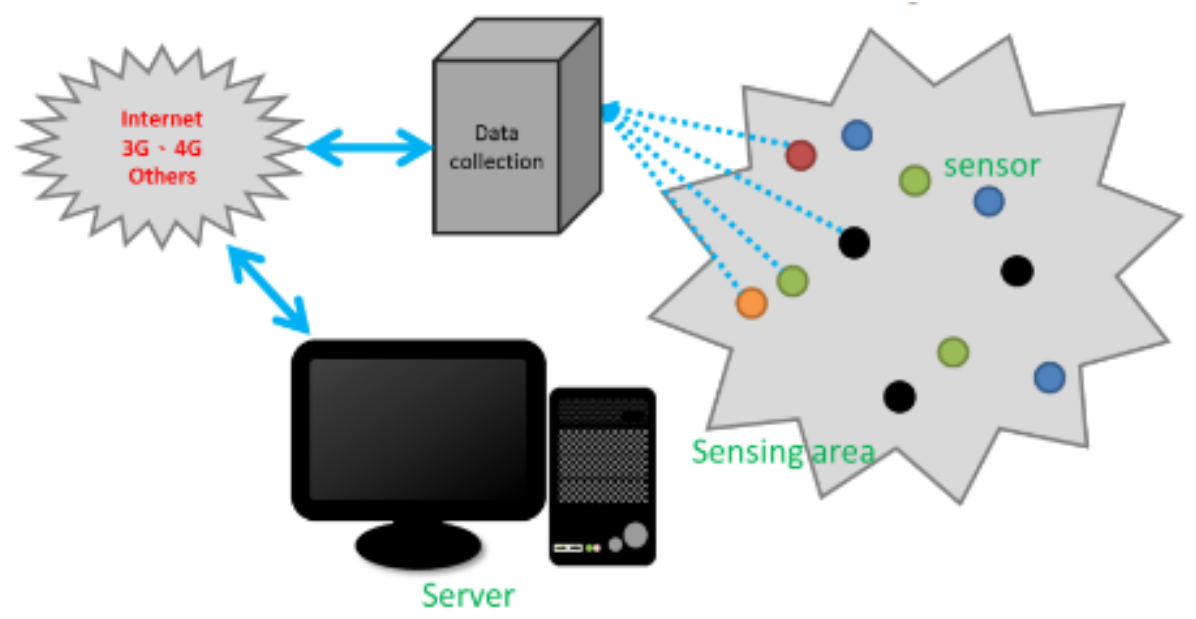

Figure. 1. The basic structure of the sensing network system

\subsection{Background - IoT}

Internet of Things (IOT) is based on Internet, traditional telecom network and other information carrier. All ordinary physical objects can be independently addressed to achieve interoperability of networks.IOTis MTM (machine to machine) with Internet. It cooperates everything constructively in the world by using RFID and wireless data communication technology. IOT generally uses a wireless network, everyone can use electronic tags to real objects Internet links and find out their specific locations. computer management and control the machine, equipment and personnel; even can remote service, cars, and search location, to prevent stolen goods. In IOT, all CPS systems can communicate with each other and pass information [6]. As shown in Figure 2, IOT can be divided into three classes:

1) Perception layer: Perception and monitoring carried out for different scenarios, with a sensing device identification and communication capabilities.

2) Network layer: The collected data from the perception layer transmitted to the Internet.

3) Application layer: According to different requirements, technology and industry expertise to develop the appropriate application integration software. 
Yang et al / Vol. 1, No. 2, December 2018, pp. 78-89

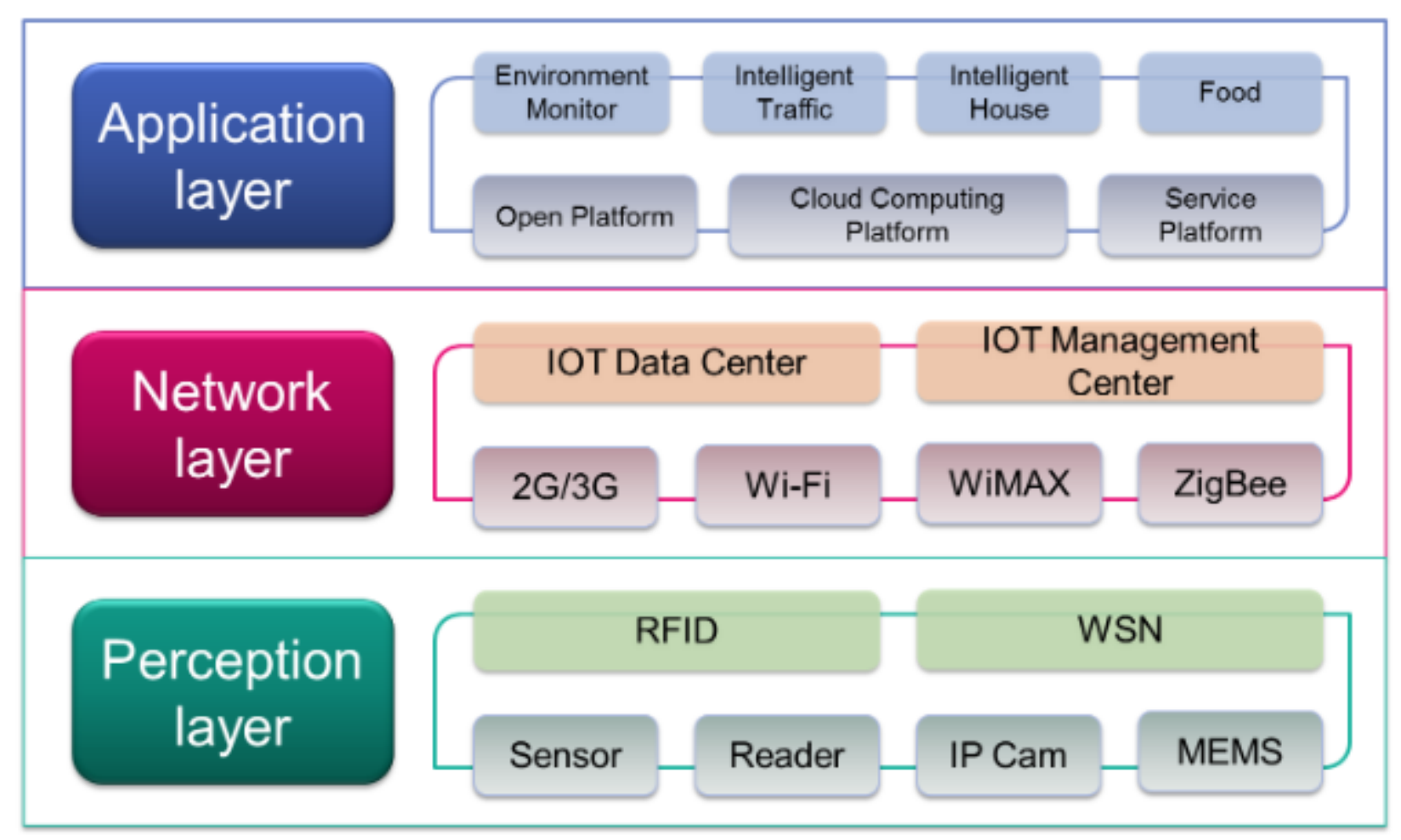

Figure. 2. Three classes of IoT.

\subsection{Background - Relational Database}

Relational database is a kind of database based on the associated model. Usea collection of algebra and other mathematical concepts and methods to deal with the information in the database. It shows the data table, each table contains a column and row. Each column of data is called record, the most representative record of the field, called the primary key column. Association model by Edgar F. Codd first proposed in 1970, and cooperate with "Codd's 12 rules". These Standard data query language SQL is a language based on relational database, retrieve and manipulate the language to perform relational database for data [8].

MySQL database system is a relational database management system (RDBMS). MySQL is a fast, multithreaded, multi-user and powerful relational database management system. Itnot only can easily connect with $\mathrm{C}, \mathrm{C}++$, Java, Perl, PHP and other languages, but also run on multiple platforms, start the service, default values for TCP / IP Port 3306 listen and applications.

\subsection{Background - Power Usage Effectiveness}

Power usage effectiveness (PUE)[11]is a metric used to determine the energy efficiency of a data center.At present,PUE is the most common international ofthe design basis and the energy assessment goalsinIT room.It is composed using the Green Grid Association rationalize energy consumption targets set by the IT room for energy.It's indicators for the IT room of energy usesettingby Green Grid Association.According to the Green Grid design proposals for planning in order to achieve optimum energy utilization. PUE is the way it calculated "total electricity consumption data center" is divided by "information center within the total electricity consumption of IT equipment".If the lower PUE value it means that the air conditioning cooling power required will be less.

\subsection{Related Work}

With the global energy supply is getting tight, plus the increasing effect of global warming. We must need to face such a severe test.To overcome the challenges attendant, it makes carbon reduction become the supreme school of learning.For information related industry personnel not only need to response the evolution of technology, but also take into consideration a number of difficulties in the global energy crunch.How to improve the management and use of the room is very important. In addition to processing performance of individual services, it has among of the largest energy-saving considerations. 
Energy regulation is one of the mostimportantsectionsin our daily life; there will be changed by using sensor tosave real-time and analysis these sensor data. In recent years, some scholars have realized the rapid processing of wireless sensor networks (WSN) with low power consumption, the use of information technology exploration of WSN environment, combined with the room's energy, the calculated value is used to calculate PUE data center energy saving standards.In the other paper by theoretical analysis and experimental test environment.ZigBee technology proven design environment monitoring system is reasonable [12]. It can be monitored by sensors placed in any position, which collected data can be analyzed. According to assessment of the necessary conditions energy based on Leadership in Energy and Environmental Design (LEED), Use above 300RTofthe central air-conditioning chiller systems in IT room,PUE value must belessthan 1.52 can meet the basic threshold of LEED.In contrast, the domestic IT room PUE average actual measurement results above about 1.9, shows that IT room with more than $30 \%$ energy-saving space.If it can integrate the use of international technology, design techniques and related standards, the new domestic IT room should be able to achieve the carbon reduction targets of LEED standard.[14]

\section{System Design and Implementation}

This section introduces the design of the proposed green energy management system and its implementation. First, a service platform in the electricity control machine room is built. Then the proposed green energy management system is implemented

\subsection{Experimental environment building}

Experimental environment In order to implement the proposed green energy management system, an experimental environment with service platform in the electricity control machine room satisfactory first built. Figure 3 (a) shows the built experimental environment. Everal electric meters we need installed in the environment and we catch data from these electric meters by using the Ethernet which transmission information to relative data library. In addition to the meters, we also introduce the air conditioning power and set temperature, humidity, and $\mathrm{CO} 2$ sensor to measure the environment information and then observe the information trend. Figure 3 (b) shows the setting of the sensors for the environment information.

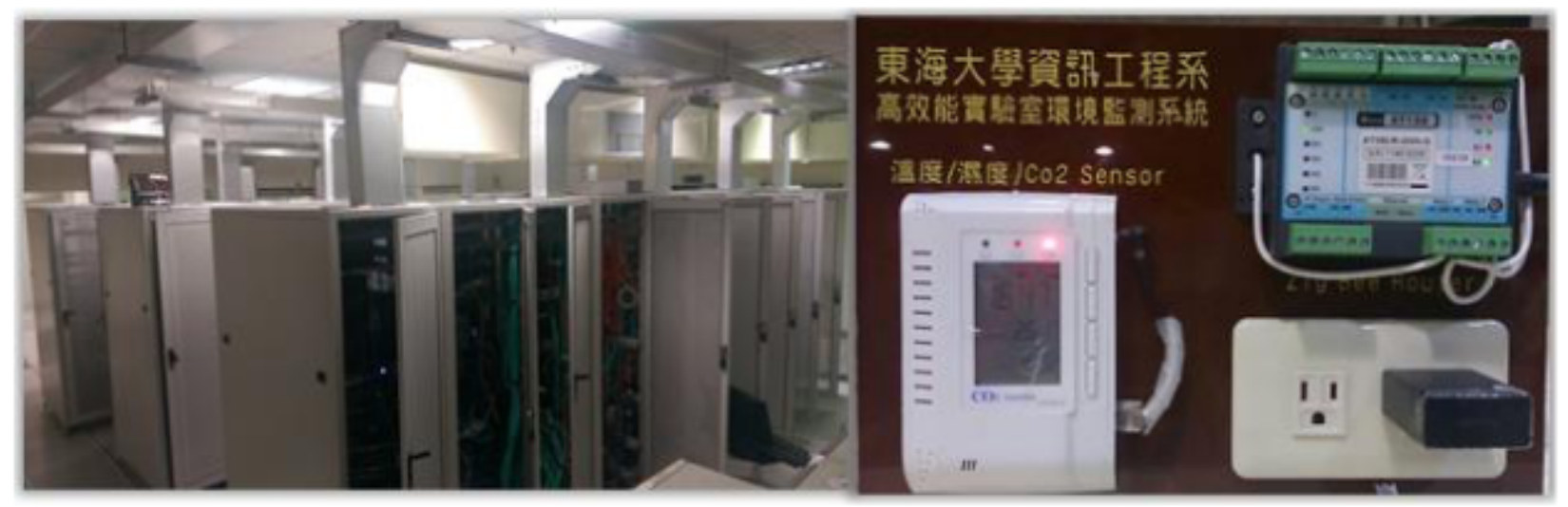

Figure. 3. (a) The built experimental environment (b) Temperature, humidity and $\mathrm{CO} 2$ sensors

\subsection{System Architecture}

For This environment monitoring system, we deployed the system onOpenStack. In Figure 4 shows that we built several virtual machines (VMs) on OpenStack. In the beginning, we set two VMs to be a Monitoringservice and Database. If the information is stored too large, we can buildmore VM to store data because OpenStack is extensible easily. We can effectively use the hardware resources of the server. 


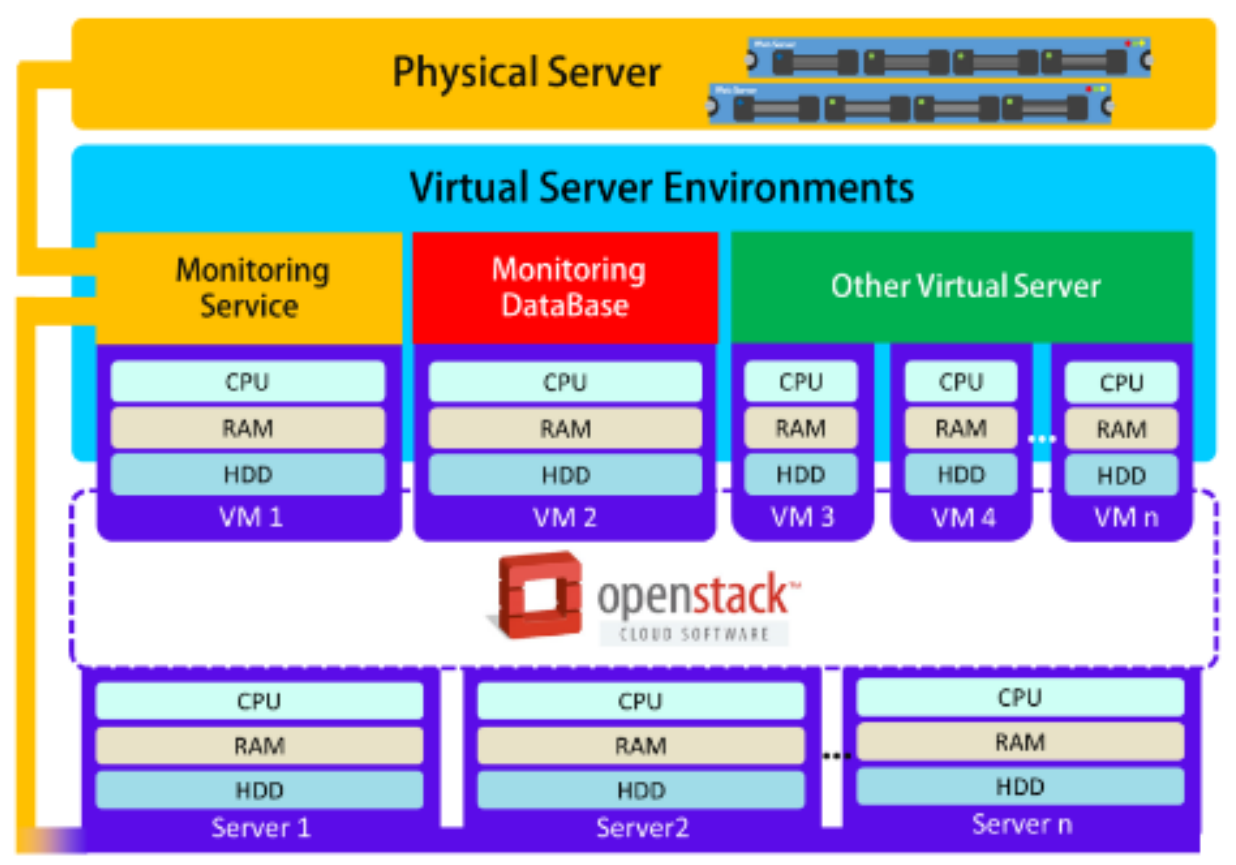

Figure. 4. System architecture base on OpenStack

Figure 5 shows our system architecture. The data from meters and sensors are transmitted to the server through the wireless network (ZigBee and internet) and stored in related database. Then, users can observe real-time information and the corresponding analysis on the interface (front-end view).

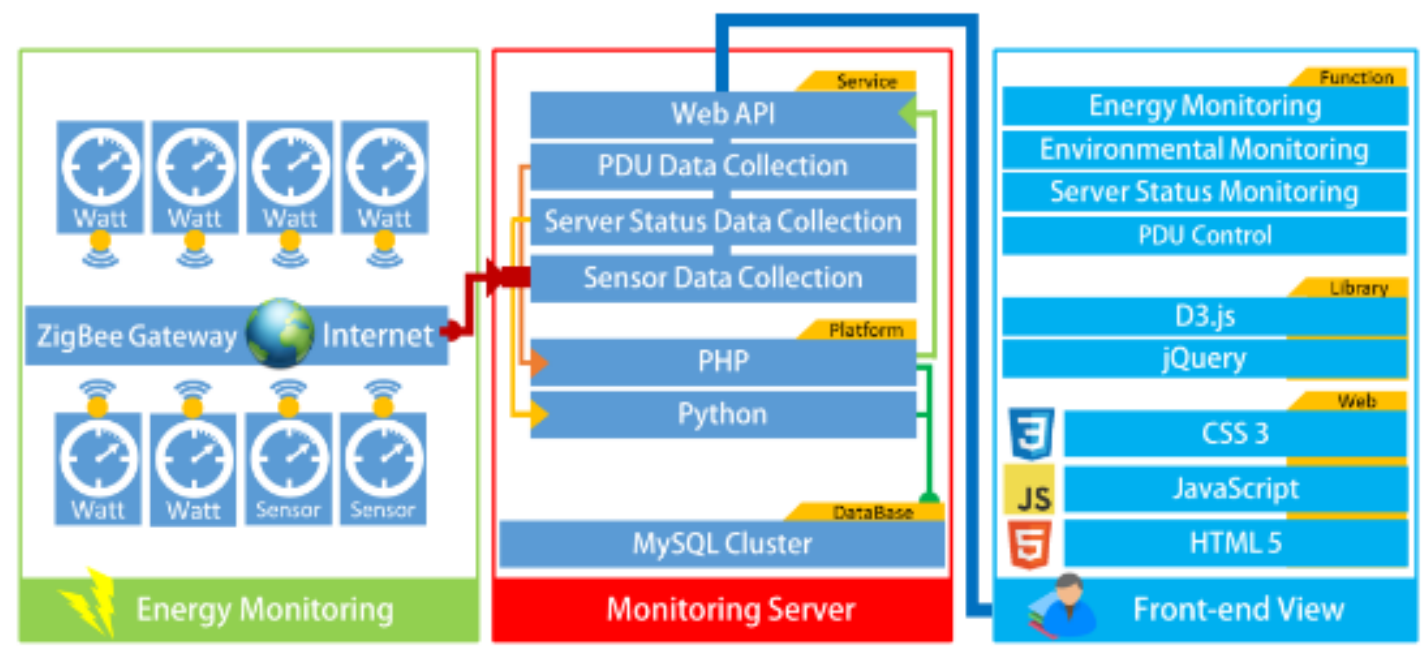

Figure. 5. Architecture of green energy management system

\section{Experimental Results}

\subsection{Authors and Affiliations}

This section, we describe our environmental monitoring System on OpenStack. Table 1 lists the hardware specification of the computer and sensors we use in the experiment. We adopt Intel Xeon 8-core E5-2640 V2@2.0GHz as the CPU of the computer. This server is the physical machine. Ubuntu 12.04 with 64 bit is adopted as our operating system. In addition, we use sensors with monitoring $\mathrm{CO} 2$, temperature, Humidity and power meters. 
Yang et al / Vol. 1, No. 2, December 2018, pp. 78-89

Table. 1. Hardware Information

\begin{tabular}{|c|c|c|c|}
\hline \multicolumn{4}{|c|}{ Hardware } \\
\hline \multirow{4}{*}{ Server } & $\mathrm{CPU}$ & \multicolumn{2}{|c|}{ Intel Xeon 8-core E5-2640 V2 2.0GHz 7.2GT/s QPI x 1} \\
\hline & RAM & \multicolumn{2}{|l|}{ 16GB RECC DDR3 $1333 \mathrm{MHz}$} \\
\hline & $\mathrm{HDD}$ & \multicolumn{2}{|c|}{ 1TB 10000RPM 3.5" SATAIII Disk x 4 (RAID 10) } \\
\hline & OS & \multicolumn{2}{|l|}{ Linux Ubuntu 12.04} \\
\hline \multirow{2}{*}{ Sensor } & \multicolumn{2}{|c|}{$\mathrm{CO} 2$, Temperature, Humidity } & ZGw08VRC \\
\hline & \multicolumn{2}{|c|}{ Wireless MULTIFUNCTION POWER METER } & WPM-100 \\
\hline
\end{tabular}

\subsection{Experimental Results}

In this section, we present three results of our power-saving information. First, we show the service which is the real-time information of all machines and the environment data in IT room. All of them will show on this interface. User can use this interface to monitor all information we obtain. Second, we calculate the PUE to help us make energy-saving base. Finally, we wrote an interface as historical information monitoring.

\subsubsection{Experimental Results}

We have established a monitoring information page of electricity. Users can survey the real-time environmental information and power information obtained from each machine. In addition, it has added a host information conditioning electricity. Through this information, we can get information from this environment and a part of power information. Access to environmental information is to make the system a better way of power saving. When user connected to the home page, the view is shown in Figure 6.

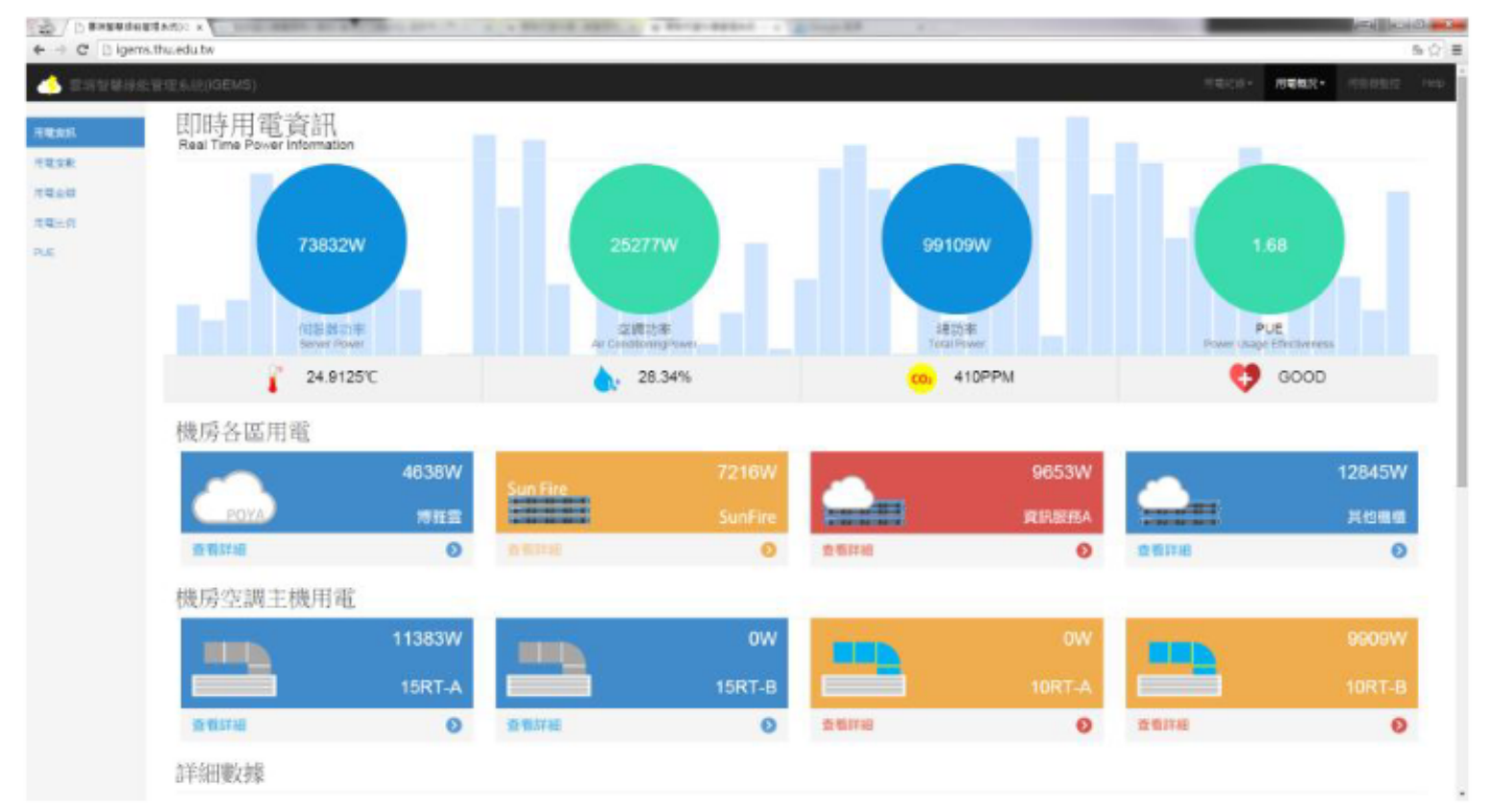

Figure. 6. Homepage of web service

As Shown in Figure 7, users can observe the detail information of voltage, current, and others. In addition, this information will update automatically per second. In Figure 8, one can see the relevant information of the temperature and humidity as well as electric power. The correlation value of cross-referencing also shows. 


\section{詳細數據}

\begin{tabular}{|c|c|c|c|c|c|c|}
\hline 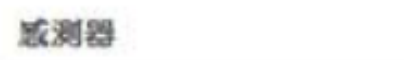 & 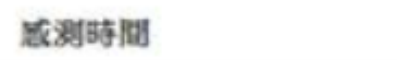 & 扈要 & 扈流 & 功宗 & 功事因数 & 颣宗 \\
\hline THUC-M0001-AC-main & $2015-02-23 \quad 16: 22: 59.418$ & 225.85 & 166.357 & 25302 & 0.68 & 60.1 \\
\hline THUC-M0002-AC-R-F & $2015-02.2316 .22 .59 .288$ & 225.85 & 75.958 & 11386 & 0.67 & 60.09 \\
\hline THUC-M0005-server-main & $2015-02-2316: 22: 59.639$ & 226.40 & 385.458 & 73162 & 0.84 & 60.1 \\
\hline THUC-M0003-AC-R-R & $2015-02-2316: 22: 59.191$ & 225.75 & 0.000 & 0 & 1.00 & 60.1 \\
\hline THUC-M0004-UPS-H & $2015-02-23 \quad 16: 22: 59.405$ & 226.45 & 64.139 & 12802 & 0.88 & 60.09 \\
\hline THUC-M0008-AC-L-RS & $2015-02-23 \quad 16: 22: 59.656$ & 226.15 & 0.000 & 0 & 1.00 & 60.1 \\
\hline THUC-M0009-AC-L-LH & $2015-02-2316: 22: 59.389$ & 225.90 & 61.761 & 9908 & 0.72 & 60,1 \\
\hline THUC-M0007-UPS-I & $2015-02-23 \quad 16: 22: 59 \cdot 242$ & 226.40 & 49.573 & 9699 & 0.86 & 60.1 \\
\hline THUC-M0011-UPS-J & $2015-02-23 \quad 16: 22: 59.37$ & 226.40 & 20.163 & 3901 & 0.85 & 60.09 \\
\hline THUC-M0012-UPS-K & $2015-02-23 \quad 16: 22: 59.38$ & 226.20 & 4.180 & 749 & 0.79 & 60.1 \\
\hline THUC-M0010-UPS-G & $2015-02-23 \quad 16: 22: 59.13$ & 226.60 & 32.015 & 4322 & 0.57 & 60.1 \\
\hline THUC-M0006-UPS-F & $2015-02-2316: 22: 59.176$ & 226.45 & 16.696 & 2945 & 0.75 & 60.1 \\
\hline
\end{tabular}

Figure. 7. Other energy-saving information screens.

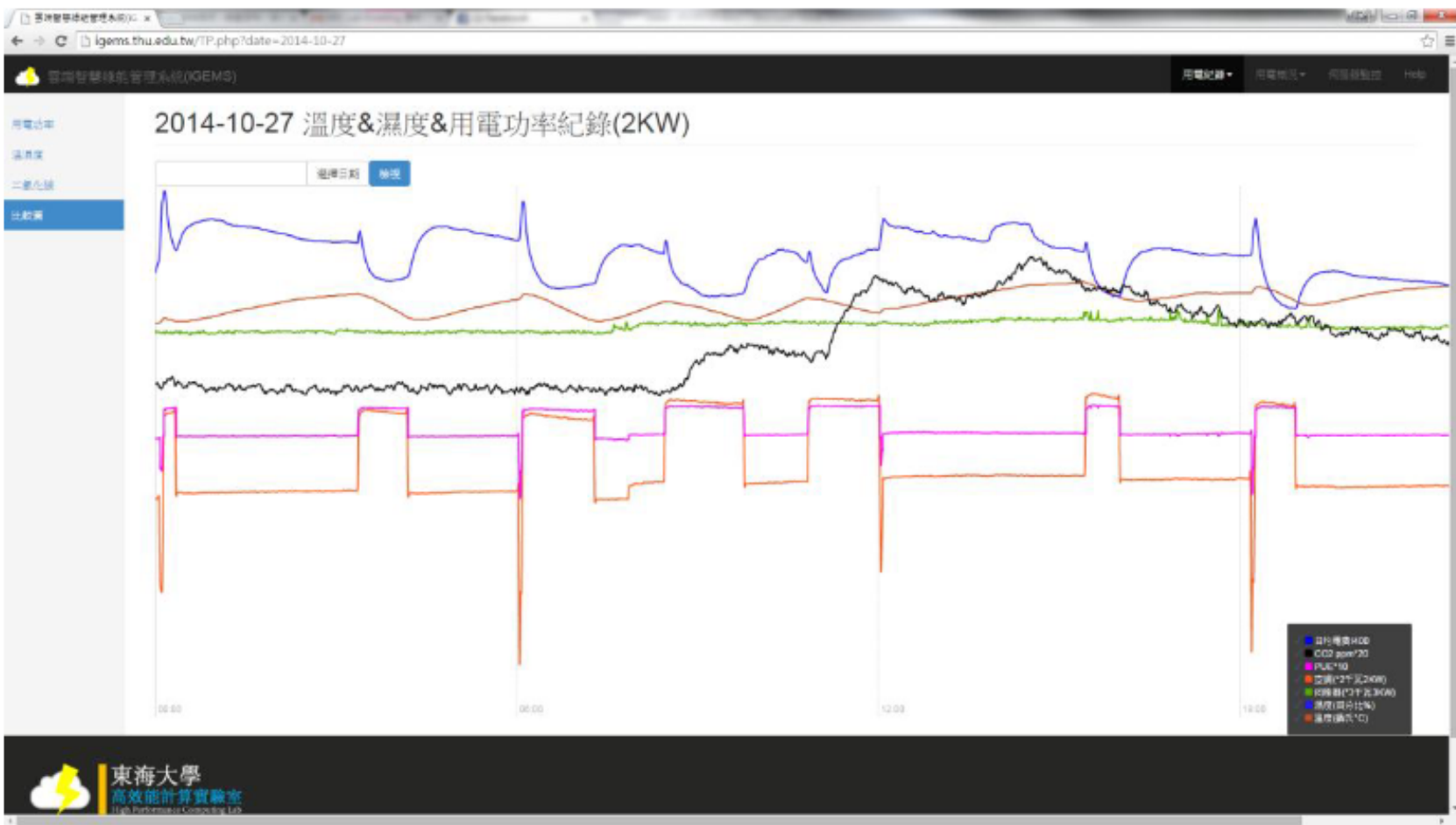

Figure. 8. All information drawn comparison chart. 


\subsubsection{Calculate PUE}

The main server room power consumption is required for the operation from the server power consumption, energy-consuming air conditioning system for cooling, power network switching equipment, and network management devices, etc. (Figure 9) For excellent energy efficiency for the server room, the energy consumption for the operation of most all servers, we calculate the PUE to help us make energy-saving basis. This study will use Power Usage Effectiveness (PUE) as energy efficiency targets of the engine room. ReducePUEvalueto assist achieve energy-saving server room. In international, the average PUE is between 1.8 and 2.1. Definition of PUE:

Totale: The total energy consumption room

ITe: IT equipment energy consumption for each machine

ACs: Air-conditioning system energy consumption for each machine

Ls: Lighting system energy consumption for each machine

E: Energy power conversion for each machine

$$
\mathrm{PUE}=\frac{\text { Totale }}{\sum \mathrm{ITe}}=\frac{\sum I T e+\sum A C s+\sum L s+\sum E}{\sum \mathrm{ITe}}
$$

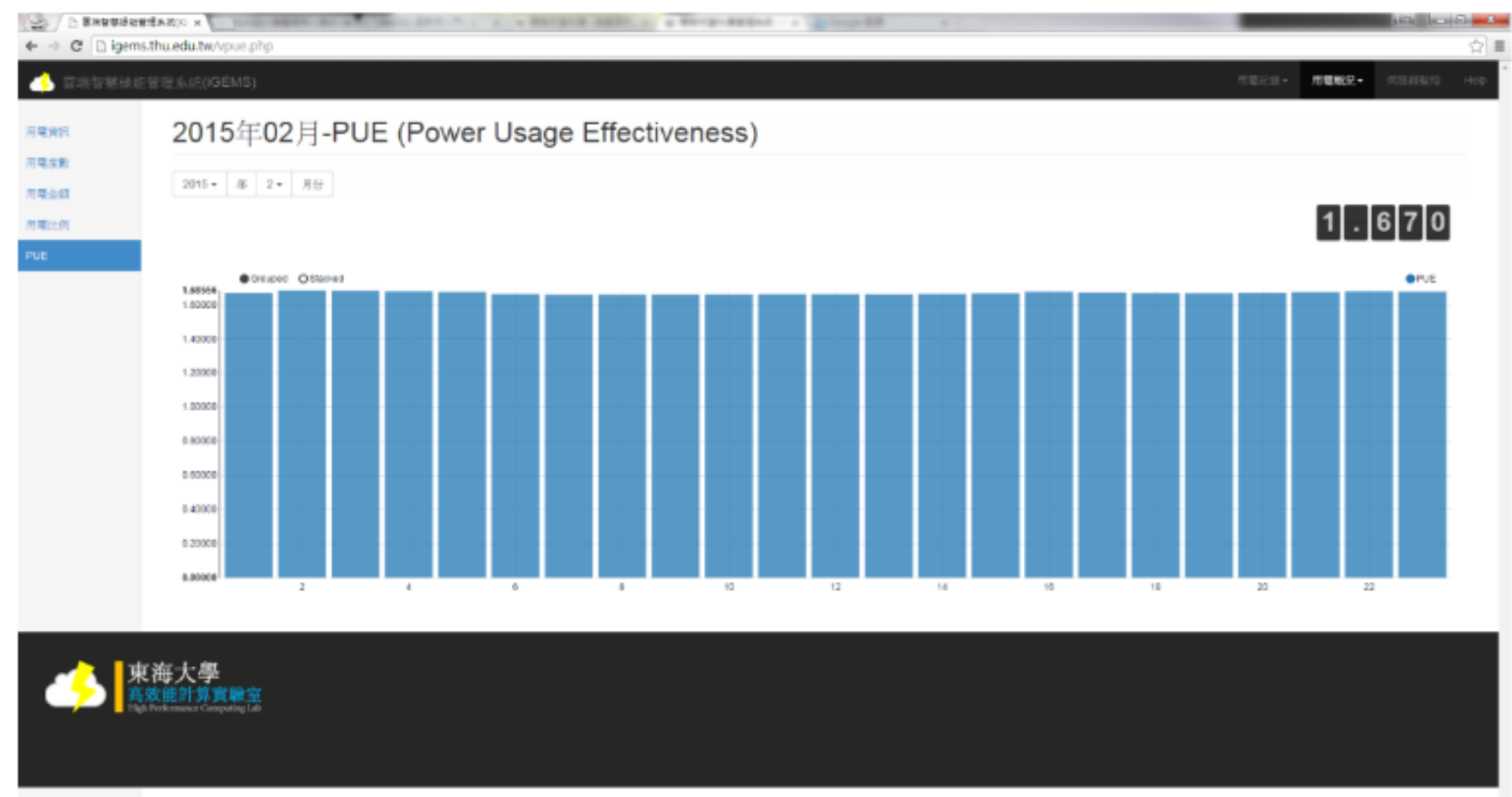

Figure. 9. CalculatePUE and show on interface

\subsubsection{Calculate PUE}

Finally, we will show the interface of our power environment monitoring for history data information. In Figure 10, through the exploration of the historical data, we can record the amount of the monthly server power, air conditioning, and power consumption each month. 


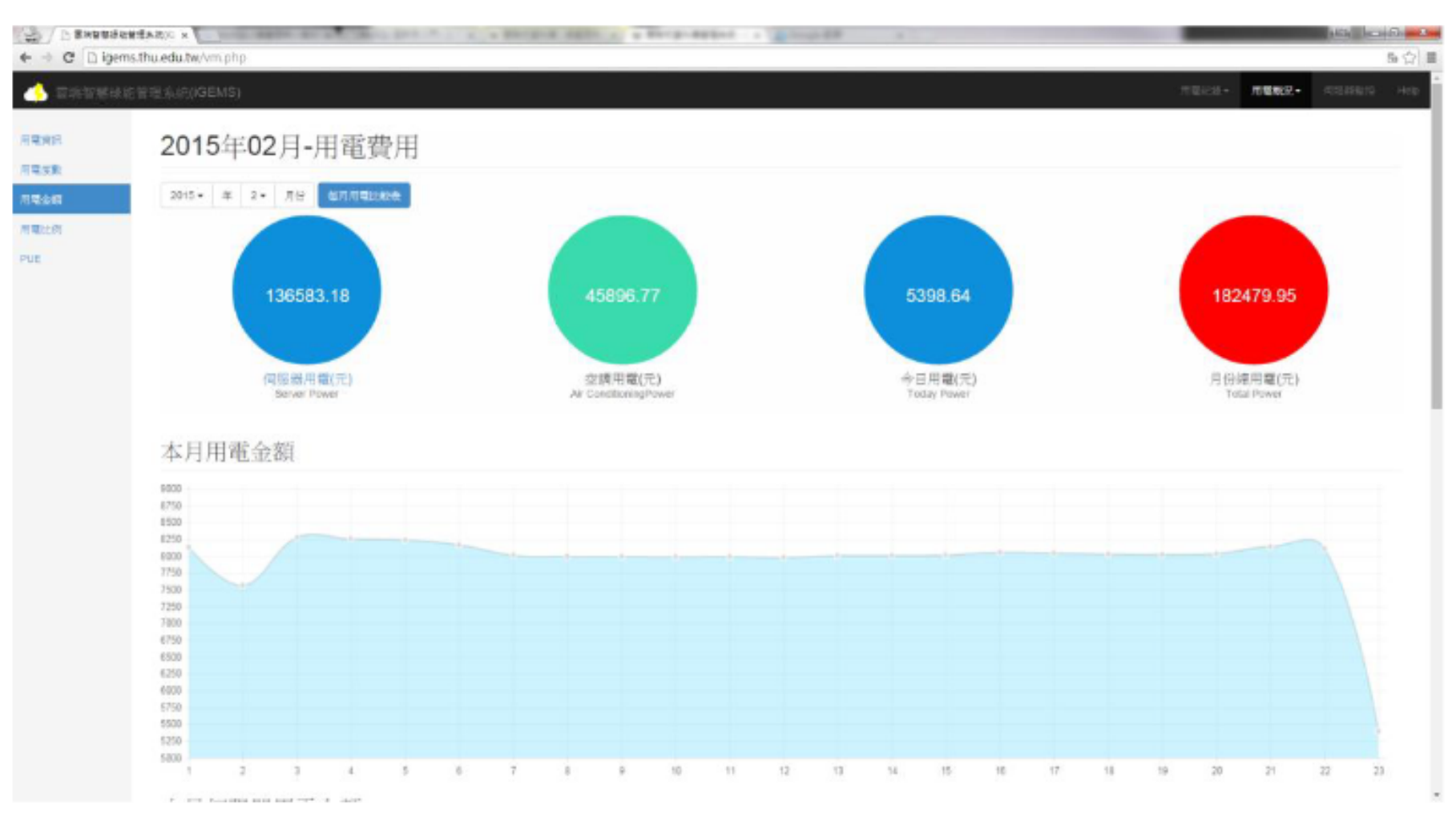

Figure. 10. The history data on interface

Due to the fact that our IT server is not closed all day long, so we can predict the electric power change this month. The data will update and calculate the average electricity costs each time when it gets a new data. It is shown in Figure 11.

\section{各月份用電比較表}

\begin{tabular}{|c|c|c|c|c|c|c|}
\hline 年/月 & 纪竝天数 & 同服器用要度数 & 空調用要度数 & 媿平費 & 日均䖳費 & PUE \\
\hline $2014-06$ & 18.42 & $30,736.040$ & $13,262.142$ & $150,473.78$ & $8,170.52$ & 1.789 \\
\hline $2014-07$ & 30.92 & $51,402.974$ & $22,751.566$ & $253,608.53$ & $8,202.97$ & 1.803 \\
\hline 2014-08 & 30.79 & $51,949.256$ & $22,421.723$ & $254,348.75$ & $8,260.31$ & 1.790 \\
\hline 2014-09 & 27.50 & $47,260.171$ & $21,632.132$ & $235,611.67$ & $8,567.70$ & 1.822 \\
\hline $2014-10$ & 30.42 & $52,339.752$ & $22,187.295$ & $254,882.50$ & $8,379.70$ & 1.780 \\
\hline $2014-11$ & 29.42 & $50,745.947$ & $20,635.988$ & $244,126.22$ & $8,298.91$ & 1.758 \\
\hline $2014-12$ & 31.00 & $54,457.880$ & $20,407.401$ & $256,039.26$ & $8,259.33$ & 1.718 \\
\hline 2015-01 & 31.00 & $55,182.497$ & $18,857.314$ & $253,216.15$ & $8,168.26$ & 1.677 \\
\hline $2015-02$ & 22.67 & $40,083.300$ & $13,470.397$ & $183,153.65$ & $8,080.31$ & 1.670 \\
\hline 本月䞍㳀 & 28 & $49,514.665$ & $16,639.902$ & $226,248.62$ & $8,080.31$ & 1.670 \\
\hline
\end{tabular}

Figure. 11. Power Cost Per month 
As shown in the records of the experiments and the proportion of electricity figure. (In Figure 12) In this room, the power consumption of air-conditioning systems is much higher than the server.

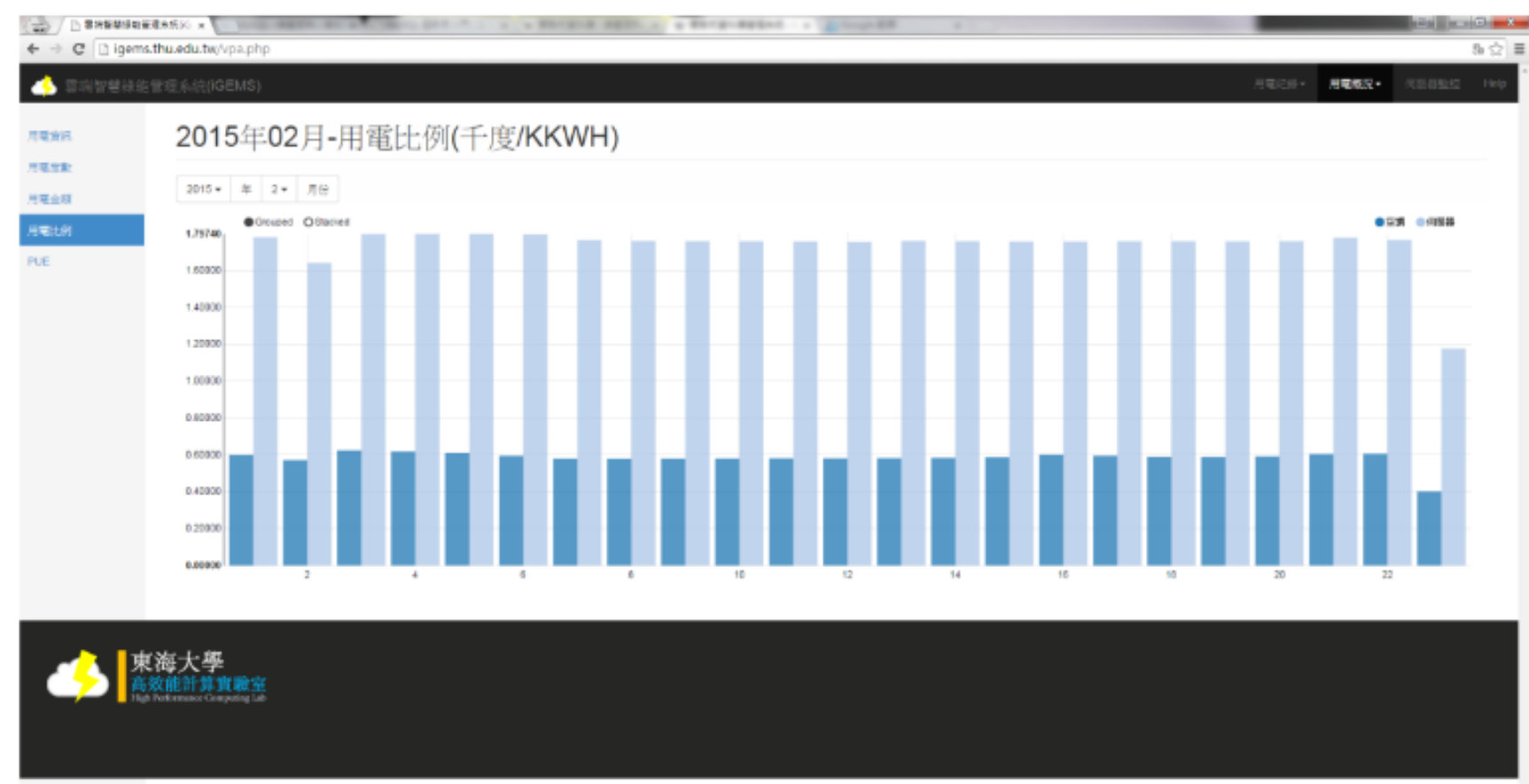

Figure. 12. The proportion of electricity

So appropriate adjust the temperature of air conditioning, allowed to decreased motor running will effectively save power consumption, achieve energy-saving effect.In Figure 13, we increase the air conditioning temperature degree setting from 26 to 27 . Then the second set of air-conditioning start frequency is reduced. This system is effective in reducing power consumption.

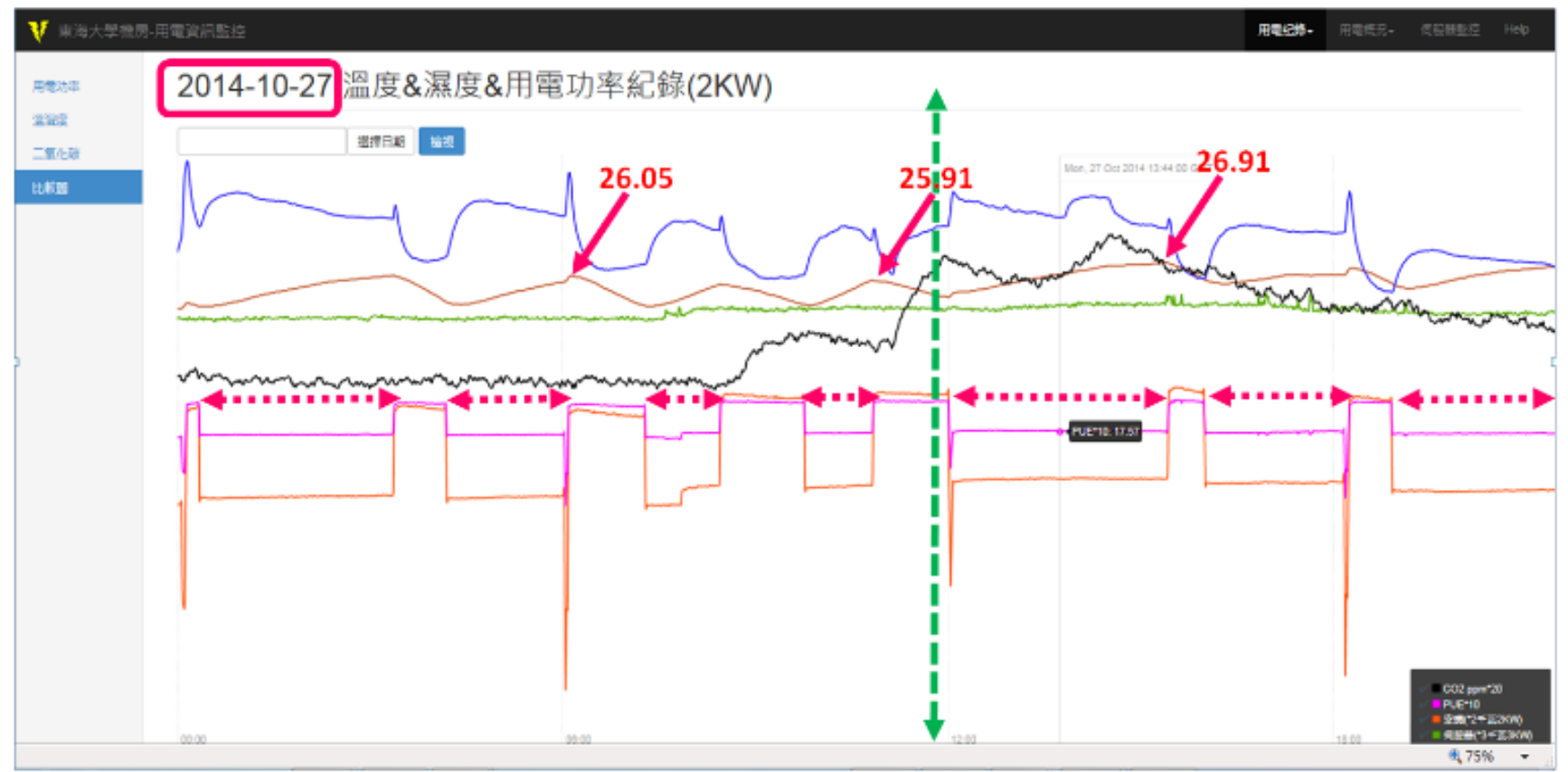

Figure. 13. Increase the air-condition to reduce power consumption 
Finally, we collect all of our data for six month which shows in Figure 14. We can clearly see that although the number of serverincrease, but during our control in temperature of air-conditions the value of PUE is in decrease and the average daily electricity is gradually reduced. It means that in our system we can find the consumption of culprit and effectively reduce energy usage.

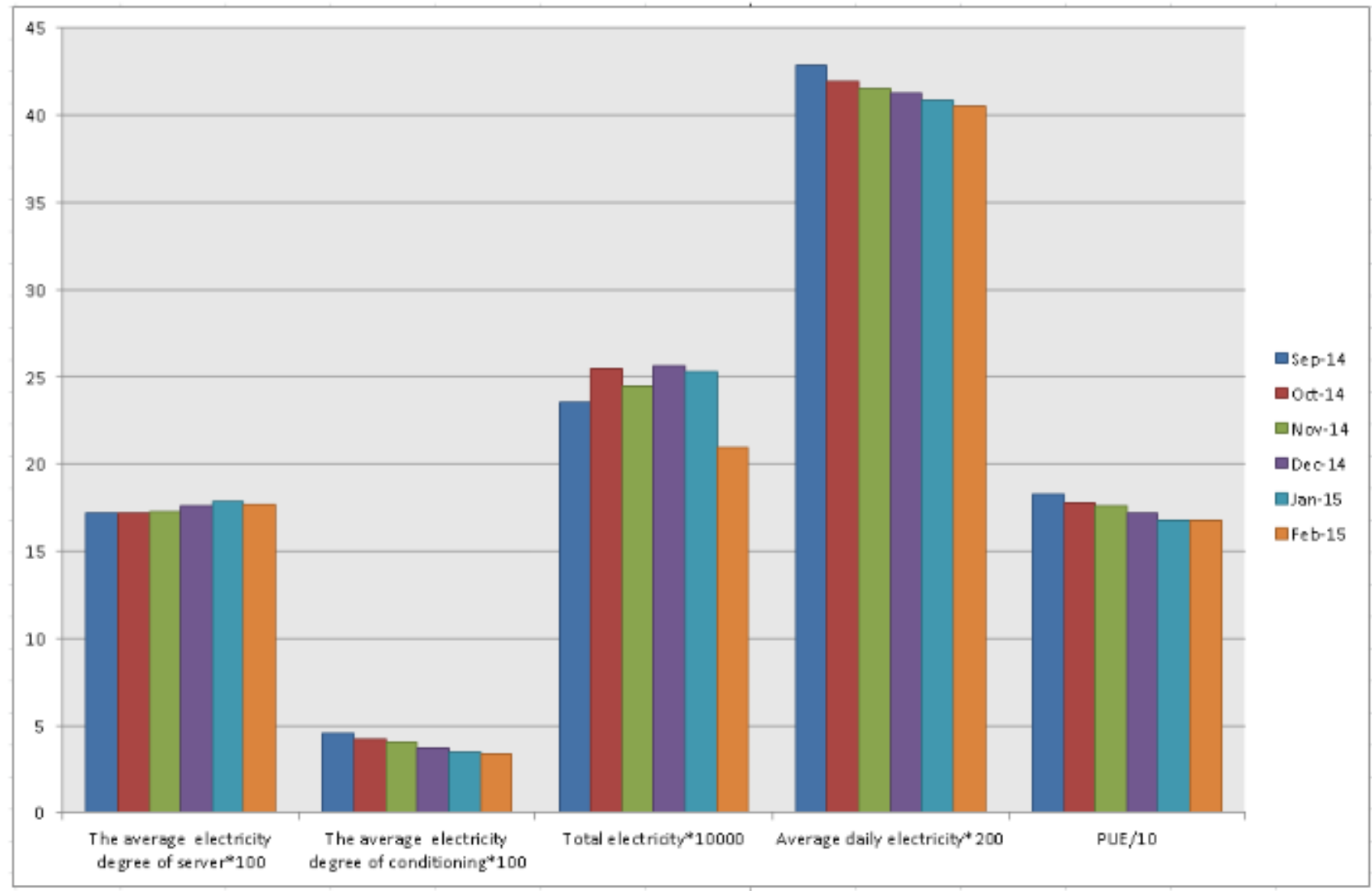

Figure. 14. The result in six months

\section{Conclusion}

The proposed green energy management system on OpenStack not only collect information of IT room to understand the situation of the energy consumption, but also obtain the degree of electricity per month, electricity tariffs, the proportion of electricity, PUE, and room environment information by user interface. The analysis of information allows us to understand the factors relative to energy consumption of ITroom.It helpsus to know how to reduce room energy consumption.

In the future, due to the amount of data increases, we will migrate the data to a distributed repository.Enhance the effectiveness of their systems. Reduce The amount of time in searching data. Furthermore Intelligence Will be added so that you can monitor and adjust theIT room information no matter where you are and what device you use.

\section{References}

[1] Wikipedia, SmartDust, http://en.wikipedia.org/wiki/Smartdust

[2] John A. Stankovic, Department of Computer Science, University of Virginia Charlottesville, "Wireless Sensor Networks" June 2006

[3] Sinem c.e. ZigBee / IEEE 802.15.4 Summary, Sep 2004.Suman Sankar Bhunia, Sarbani Roy, and Nandini Mukherjee. Iems: Indoor environment monitoring system using zigbee wireless sensor network. In Proceeding ICCCS'11 Proceedings of the 2011 International Conference on Communication, Computing \& Security, pages 142-145, 2011. 
Yang et al / Vol. 1, No. 2, December 2018, pp. 78-89

[4] Shun-qi Chai, Lei Ji, and Hong Wu. Zigbee wireless sensor network for environmental monitoring system. pages 75081R $75081 \mathrm{R}-6,2009$

[5] National Electrical Manufacturers Association, "American National Standard Protocol Specification For Interfacing to Data Communication Networks", January 9, 2009

[6] Tarry Singh, Pavan Kumar Vara "Smart Metering the Clouds" 2009 18th IEEE International Workshops on Enabling Technologies: Infrastructures for Collaborative Enterprises

[7] Wenbin Jiang, Hao Li, Hai Jin, Lei Zhang, Yaqiong Peng, "VESS: An Unstructured Data-Oriented Storage System for Multi-Disciplined Virtual Experiment Platform", Proceedings of the International Conference on Human-centric Computing 2011 and Embedded and Multimedia Computing 2011.

[8] Divyakant Agrawal, Amr El Abbadi, Sudipto Das, Aaron J. Elmore "Database Scalability, Elasticity, and Autonomy in the Cloud", 16th International Conference, DASFAA 2011, Hong Kong, China, April 22-25, 2011, Proceedings, Part I .

[9] Feng Zhu, Jie Liu, Lijie Xu "A Fast and High Throughput SQL Query System for Big Data", 13th International Conference, Paphos, Cyprus, November 28-30, 2012. Proceedings.

[10] MySQL Cluster Architecture Overview. A mysql®technical white paper. April, 2004.

[11] Victor Avelar, Schneider Electric Dan Azevedo, Disney Alan French, Emerson Network Power, PUE Tм: A COMPREHENSIVE EXAMINATION OF THE METRIC, 2012

[12]H. Dae-Man and L. Jae-Hyun, "Design and implementation of smart home energy management systems based on zigbee," IEEE Transactions on Consumer Electronics, vol. 56, pp. 1417-1425, 2010.

[13] P. Wan-Ki, C. Chang-sic, L. Il-woo, and J. Jonghyun, "Energy efficient multi-function home gateway in always-on home environment," IEEE Transactions on Consumer Electronics, vol. 56, pp. 106-111, 2010.

[14] I.K. Yang, N.J. Jung, Y.I. Kim, "Status of Advanced Metering Infrastructure Development in Korea", Korea Electric Power Research Institute IEEE T\&D pp. 1-3, 26-30 Asia 2009 